\title{
ENGEVISTA
}

Página da revista: http://www.uff.br/engevista/seer/

\section{Avaliação da perda de massa de colmos de bambu durante o processo de secagem}

\section{Evaluation of the mass loss of bamboo stalks during the drying process}

\author{
Cassiano Rodrigues Moura ${ }^{1}$ \\ Luiz Veriano Oliveira Dalla Valentina ${ }^{2}$ \\ Adalberto José Tavares Vieira ${ }^{3}$
}

Resumo: A fibra de bambu vem sendo amplamente utilizada como reforço em matrizes estruturais e materiais compósitos. A primeira etapa do processamento deste produto é a cura, um processo de secagem que geralmente é realizado em estufas e contribui para melhorar suas propriedades mecânicas. O objetivo deste trabalho é avaliar o processo de secagem de colmos de bambu e definir as melhores condições para se alcançar uma fibra com baixo teor de umidade. A metodologia de pesquisa utilizada neste trabalho é de caráter literário e experimental. A planta investigada é da espécie Bambusa Vulgarise, que foi seca sobre temperatura controlada. Estas foram avaliadas quanto à remoção de extrativos e do cálculo de perda de massa. Os colmos de bambu apresentaram uma perda de umidade média de $47 \%$ que varia conforme a espessura dos colmos. Pode-se constatar que o tempo de secagem para se alcançar fibras com teor de umidade inferior a $0,5 \%$ é maior que $14 \mathrm{~h}$. Estes resultados serão apresentados e discutidos evidenciando sua influência para a cadeia produtiva da fibra de bambu.

Palavras-chave: Fibra de bambu; Perda de massa; Secagem; Bambusa Vulgarise.

\begin{abstract}
Bamboo fiber has been widely used as reinforcement in structural matrices and composite materials. The first step in the processing of this product is curing, a drying process that is usually carried out in greenhouses and contributes to improve its mechanical properties. The objective of this work is to evaluate the drying process of bamboo stalks and to define the best conditions to reach a fiber with low
\end{abstract}

\footnotetext{
${ }^{1}$ IFSC - Instituto Federal de Santa Catarina

${ }^{2}$ UDESC - Universidade do Estado de Santa Catarina

${ }^{3}$ UDESC - Universidade do Estado de Santa Catarina
}

ENGEVISTA, V. 20, n.3, p.476-486, Julho 2018. 
ISSN: $1415-7314$

ISSN online: 2317-6717

moisture content. The research methodology used in this work is literary and experimental. The investigated plant is of the species Bambusa Vulgarise, which was dried under controlled temperature. These were evaluated for the removal of extractives and the calculation of mass loss. The bamboo stems had a mean moisture loss of $47 \%$, which varies according to the thickness of the stalks. It can be observed that the drying time to reach fibers with a moisture content of less than $0.5 \%$ is greater than $14 \mathrm{~h}$. These results will be presented and discussed evidencing their influence on the bamboo fiber productive chain.

Keywords: Bamboo fiber; Weight loss; Drying; Bambusa Vulgaris. 


\section{Introdução}

Pesquisas sobre fibras vegetais e suas aplicações na manufatura de componentes construtivos, podem ainda ser consideradas como escassas e recentes, apesar das vantagens que esse tipo de material apresenta sobre as demais.

A utilização do bambu, bem como de sua fibra, vem crescendo no mercado tanto brasileiro como mundial. Com o decréscimo da oferta de recursos naturais e grande desenvolvimento da indústria tem-se buscado saídas alternativas e sustentáveis para se contribuir com o meio ambiente.

O uso da fibra do bambu para construção de placas de concreto, substituindo o aço, já se mostrou viável, diversos autores já vem estudando essas e outras aplicações (GHAVAMI, 2005; XIE XIet al., 2015; KONGKEAW et al., 2011).

Segundo Liu et al., (2012) as fibras vegetais naturais têm contribuído de forma positiva para a prosperidade econômica e a sustentabilidade em nossas vidas diárias. Especificamente a fibra de bambu que vem sendo utilizadas para aplicações industriais como processos têxteis, indústrias de papel e construção civil. O bambu vem sendo estudado como um dos materiais alternativos na construção civil devido a sua grande resistência à tração, facilidade no plantio e rapidez de crescimento.

As fibras são materiais que possuem geometria uniforme e grandes comprimentos em relação ao seu diâmetro, sendo que sua função varia de acordo com suas propriedades físicoquímica.

Para aplicação como reforço em matrizes cimentícias uma das etapas de seu processamento é a secagem. Conforme Deus et al., (2005) após esse processo, as fibras tem um aumento considerável na sua resistência a flexão, quando comparadas ao material in natura. Diante do exposto o objetivo do presente trabalho é avaliar e definir as melhores condições de secagem para as fibras de bambu da espécie Bambusa Vulgarise.

\section{Fundamentação teórica}

\subsection{Bambu - aspectos botânicos}

Conforme Nogueira (2009), bambu é o nome que se dá às plantas da subfamília bambusoideae, da família das gramíneas (Poaceae ou Gramineae). Segundo Reis (2013), encontram-se espalhadas pelo mundo aproximadamente 50 gêneros e 1500 espécies dessa planta. Diversas destas espécies são utilizadas como aplicações estruturais em casas, pontes, prédios e plataformas de construção (CHUNG e YU, 2002).

Genericamente a planta, da mesma forma que as árvores, apresentam uma parte aérea constituída pelo colmo, folhas e ramificações e outra subterrânea composta pela raiz, rizoma e brotos. A parte aérea conhecida como tronco, ou caule das árvores, é denominado colmo, sendo 
normalmente oco. O eixo central cilíndrico é formado por nós e entrenós variando de uma espécie para outra (YUMING e CHAOMAO, 2010).

Os colmos apresentam diâmetro regular com seção definida, são separados por paredes transversais conhecidos como diafragmas, que separam as câmaras ocas da planta, conforme se pode observar na Figura 1.

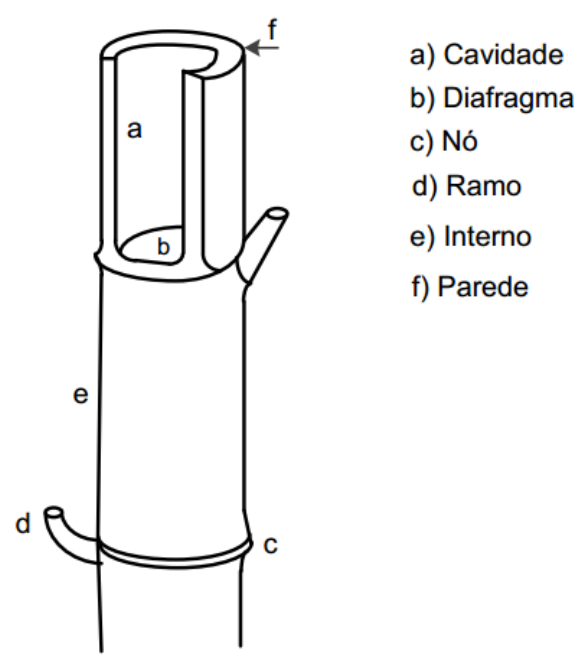

Figura 1. Anatomia do colmo.

Fonte: Adaptado de Pereira (2001)

\subsection{Fibras naturais}

As fibras são materiais que possuem geometria uniforme e grandes comprimentos em relação ao seu diâmetro, sendo que sua função varia de acordo com suas propriedades físicoquímica. Cada tipo de fibra possui características particulares e o seu aproveitamento como matéria prima baseia-se em suas propriedades, como a capacidade de alongamento, resistência, densidade, disponibilidade, entre outros.

Segundo Persson et al., (1984), pode-se classificar as fibras em naturais ou sintéticas, conforme mostra a Figura 2. A fibra de bambu nesta classificação seria uma ramificação das fibras de origem vegetal. 


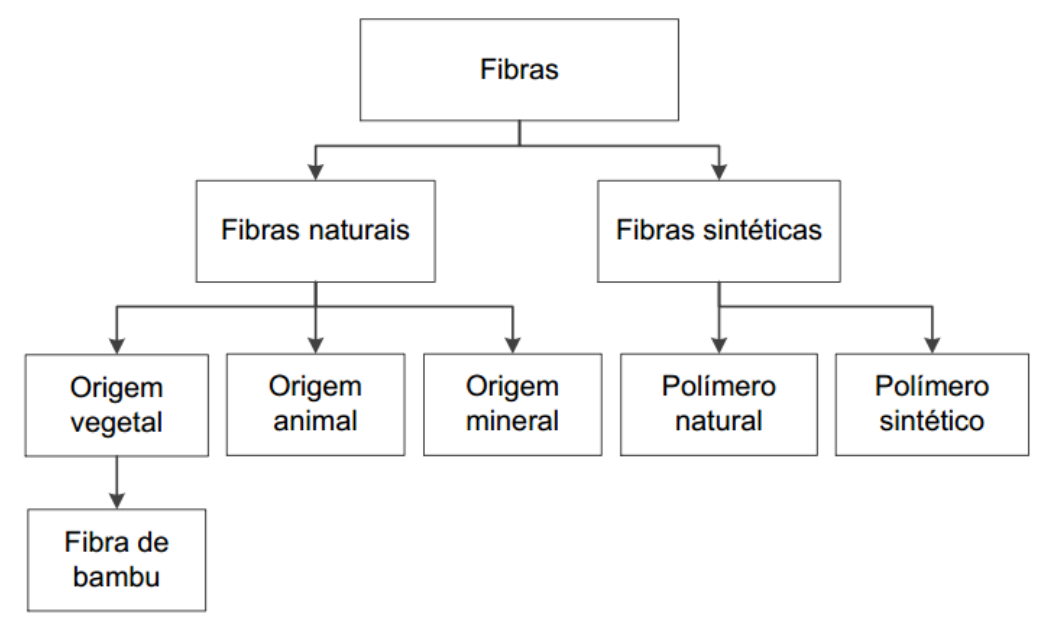

Figura 2. Classificação dos tipos de fibras.

Fonte. Adaptado de Persson et al., (1984).

A estrutura básica da fibra é composta por hemicelulose, celulose, lignina, além de constituintes com menores percentuais como pectina, sais inorgânicos, substâncias nitrogenadas e corantes naturais (PHILIPP e D'ALMEIDA, 1998). Conforme Bledzi, Gassan (1999), as fibras vegetais, conhecidas como lignocelulosicas, em comparação com as sintéticas, apresentam diversas vantagens em relação a sua utilização.

Este fato se deve a características como origem natural abundante, baixo custo, fácil renovação, baixa densidade, propriedades específicas, não toxicas e biodegradáveis. Além de contribuírem para a redução do efeito estufa devido à liberação de $\mathrm{CO}_{2}$ durante seu cultivo.

Cada fibra vegetal é constituída de várias fibras elementares, ligadas por um material de cementação constituído basicamente de lignina. A característica de cada componente das fibras vegetais é descrita a seguir:

a) CELULOSE - Principal componente da parede celular da fibra vegetal. É um polissacarídeo da família dos carboidratos. De acordo com Machado (2000), esse componente para o bambu é de 40 a 50\%;

b) HEMICELULOSE - É uma mistura de vários polímeros polissacarídeos de baixa massa molecular, que estão ligados com a celulose nos tecidos vegetais (CABALLERO E MARCILLA, 1996);

c) LIGNINA - Constitui a fração não contêm carbono da fibra, principal componente da camada intercelular. Funciona como elemento de suporte oferecendo resistência ao impacto, compressão e dobra (FRANCO, 2010). 


\section{Material e métodos}

A metodologia de pesquisa utilizada neste trabalho é de caráter literário e experimental. A Figura 3 apresenta o fluxo para o procedimento experimental utilizado nesta pesquisa. Pode-se observar que o processo se inicia com a seleção do bambu e finaliza com a verificação da perda de massa.

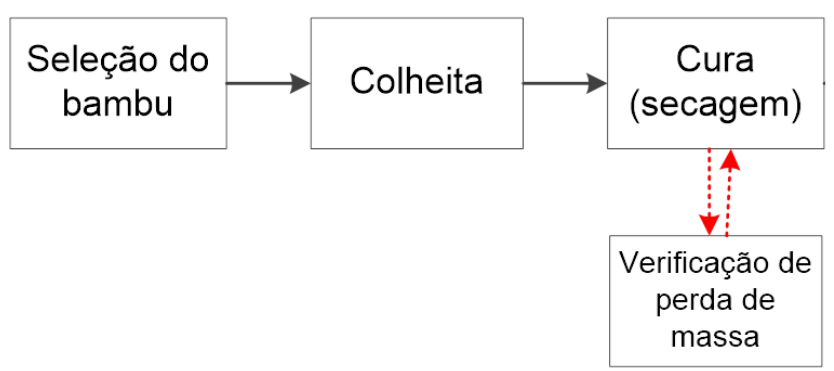

Figura 3. Procedimento experimental utilizado nesta pesquisa.

A espécie de bambu utilizada foi a Bambusa Vulgarise. A planta foi fornecida pela empresa ENVILLE, localizada na região de Joinville/SC. Conforme recomendado por Reis (2013) a planta foi colhida com idade de aproximadamente 5 anos, período médio onde ela apresenta maior resistência nos colmos.

Foi utilizado o terceiro entrenó da planta para as análises experimentais devido às mudanças nas propriedades mecânicas ao longo da disposição dos colmos conforme é mostrado na Figura 4.

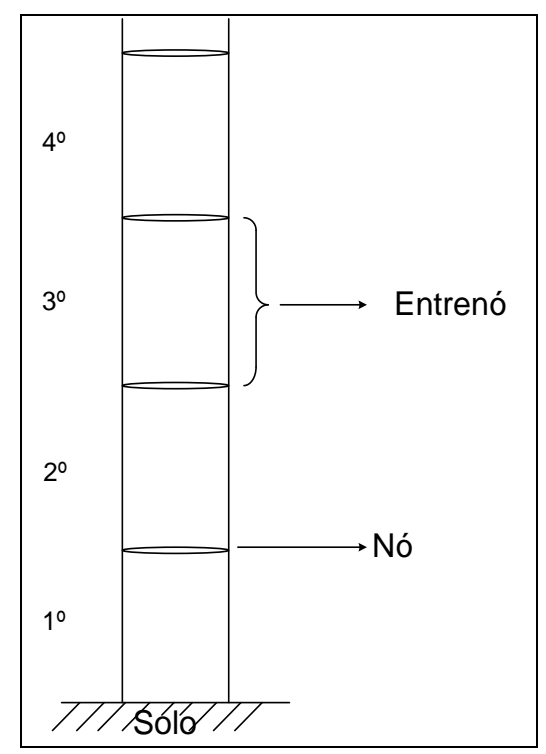

Figura 4. Seleção do colmo. 
Após a retirada do local os bambus foram cortados e medidos com auxílio de escala graduada e paquímetro, conforme mostra a Figura 5. Foram analisados cinco colmos recémcortados (amostras), esses foram pesados com auxílio de uma balança digital de precisão semianalítica e os valores registrados em tabela.

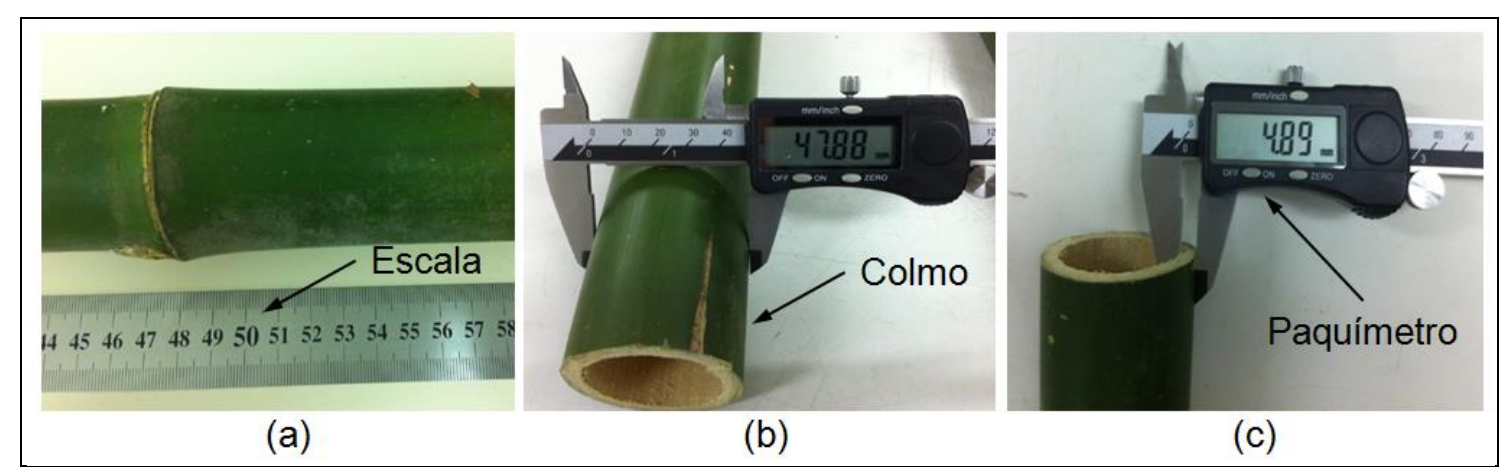

Figura 5. Medição dos colmos - (a) comprimento, (b) diâmetro, (c) espessura.

Após o detalhamento técnico do colmo foi realizado o processo de cura, com auxílio de uma estufa da empresa Quimis, modelo Q314D-243. O material foi aquecido a uma temperatura de $100^{\circ} \mathrm{C}$, conforme realizado por Reis (2013). O percentual de umidade foi monitorado hora/hora até o momento em que as variações das massas se apresentaram inferiores a $0,5 \% \mathrm{o}$ que se considerou desprezível. Ao final o material foi resfriado a temperatura ambiente. Posteriormente foi realizado o cálculo percentual do teor de umidade (m), através da Equação (1).

$$
\mathrm{m}(\%)=\frac{\mathrm{m}_{\mathrm{i}}-\mathrm{m}_{\mathrm{f}}}{\mathrm{m}_{\mathrm{i}}} \times 100
$$

Onde:

— m é o teor de umidade percentual;

- $\mathrm{m}_{\mathrm{i}}$ é a massa inicial em gramas;

- $\mathrm{m}_{\mathrm{f}}$ é a massa em gramas medida após um período de tempo.

\section{Resultados e Discussão}

O detalhamento técnico e as informações referentes ao bambu utilizado são apresentados na Tabela 1.

Tabela l. Informações técnicas da planta.

\begin{tabular}{ll}
\hline Bambu & Características \\
\hline Espécie & Bambusa Vulgarise \\
Idade & 5 anos \\
Altura & $18 \mathrm{~m}$ \\
\hline Dados do Terceiro entrenó & Características \\
\hline
\end{tabular}




\begin{tabular}{ll}
\hline Espessura média de parede & $6,22 \mathrm{~mm}$ \\
Diâmetro médio & $48,95 \mathrm{~mm}$ \\
Comprimento médio & $476 \mathrm{~mm}$ \\
\hline
\end{tabular}

A aparência dos colmos pode ser observada na Figura 6, onde se observa o bambu innatura (a) e os colmos após o processo de secagem (b).

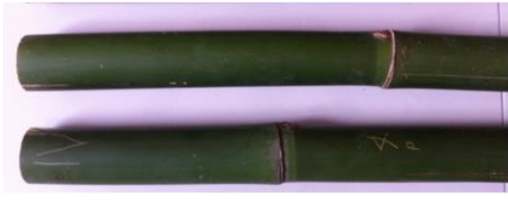

(a)

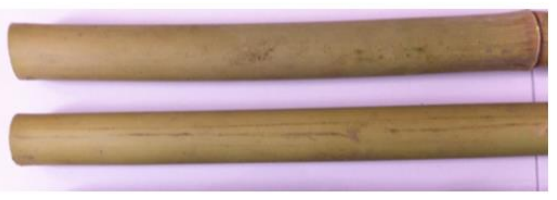

(b)

Figura 6. Aparência dos colmos de bambu antes (a) e depois (b) do processo de secagem

A Figura 7 apresenta as curvas de secagem referentes às amostras (A, B, C, D, E) das fibras de bambu obtidas com a aplicação do processo de cura. Pode-se observar que na fase inicial do processo ocorre uma perda massa acentuada devido a grande quantidade de liquido presente nos colmos, isto é uma característica de bambus com essa idade. Esse nível de perda de massa tende a cair conforme se prolonga o tempo de secagem.

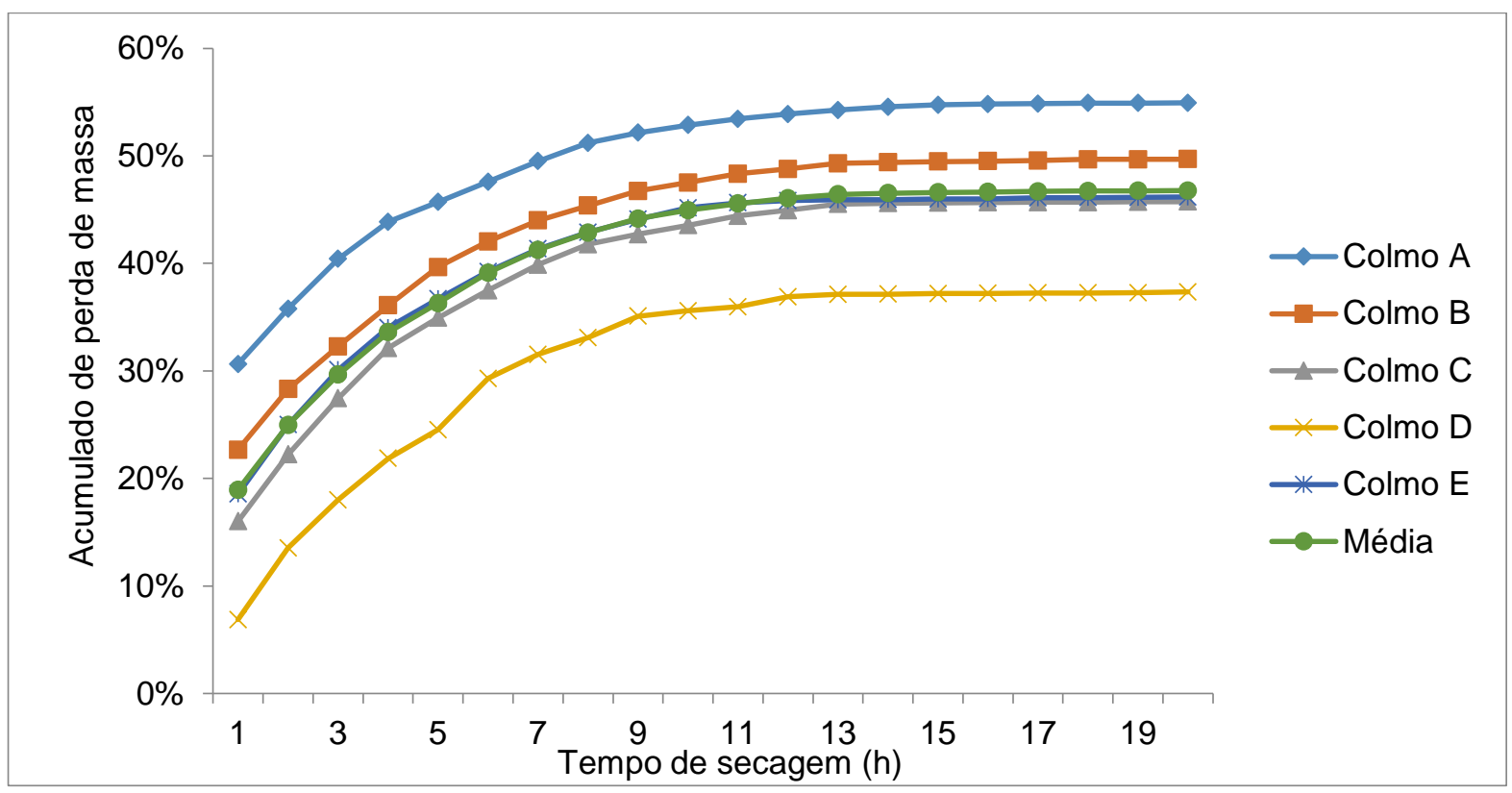

Figura 7. Curva de secagem das fibras de bambu.

A Figura 8 apresenta a média percentual de perda de umidade no processo de secagem. Pode-se constatar por essa curva que aproximadamente em $14 \mathrm{~h}$ o processo de secagem alcança um nível linear, tornando a perda de massa quase que imperceptível. Essa perda de umidade nesse momento é $0,1 \%$ em média, ou seja, muito próxima à zero. 


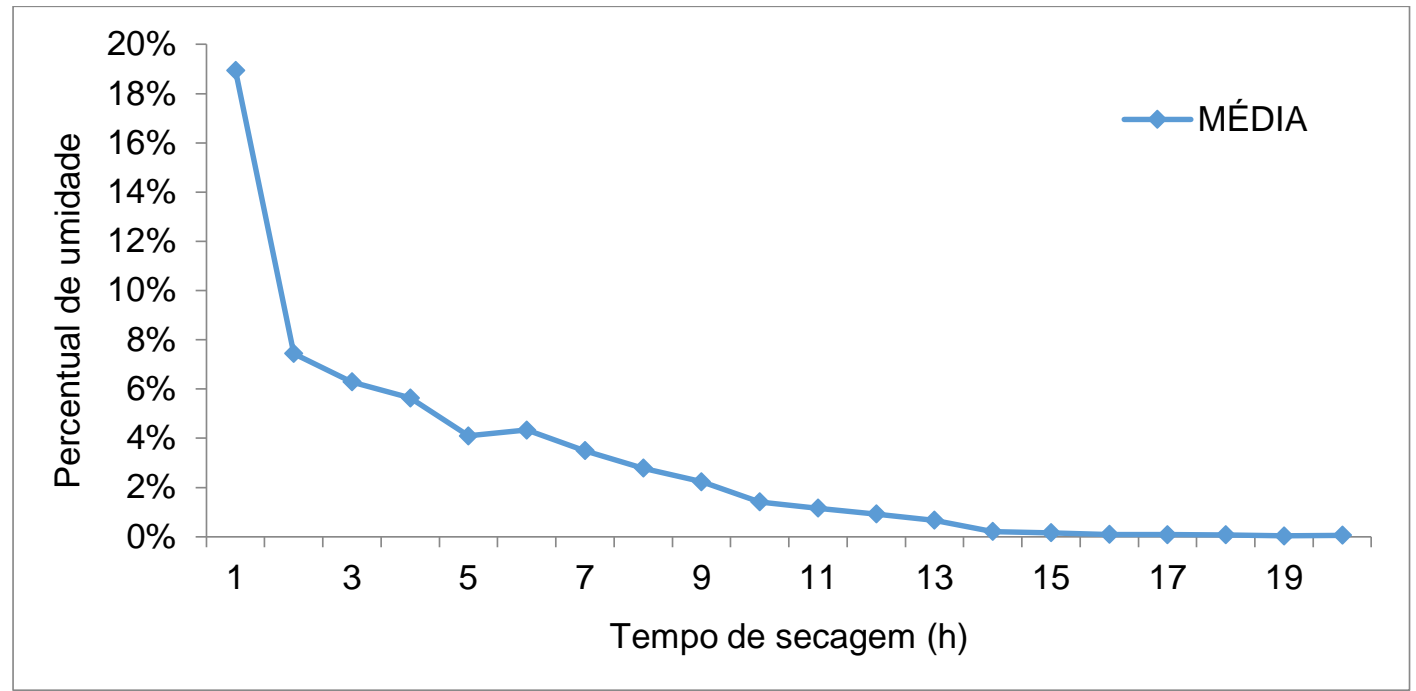

Figura 8. Média percentual de perda de umidade no processo de secagem.

A perda de massa em média pode chegar a $47 \%$ durante o processo de secagem, conforme se pode observar na Figura 9, isso pode variar de acordo com a espessura do colmo da planta. Esse percentual é retirado do bambu na forma de umidade.

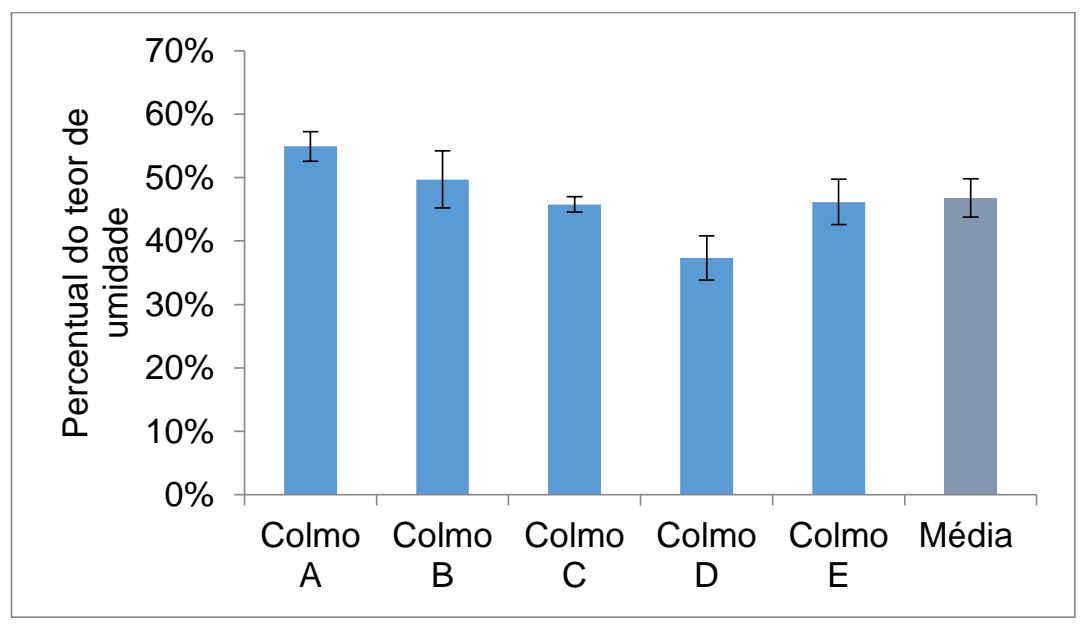

Figura 9. Percentual de perda de massa dos bambus no processo de secagem.

\section{Conclusões}

Com a aplicação do processo de secagem dos colmos foi possível constatar que o tempo médio para se alcançar um teor de umidade abaixo de $0,5 \%$ é de $14 \mathrm{~h}$, onde a perda de massa pode-se considerar imperceptível. Foi comprovado que aproximadamente $47 \%$ da massa dos colmos são retiradas na forma de umidade durante o processo de secagem, o que pode variar de acordo com a espessura da planta.

De posse destes parâmetros pode-se contribuir para melhorar a qualidade do 
processamento das fibras de bambu, uma vez que o processo de secagem (cura) faz parte das etapas iniciais da cadeia produtiva deste produto.

\section{Agradecimentos}

Agradecimento Ao Programa de Pós-Graduação em Ciências e Engenharia de Materiais da UDESC/CCT pela realização dos experimentos no Laboratório de materiais e a empresa ENVILLE pelo fornecimento das amostras.

\section{Referências}

BLEDZKI, A. K.; GASSAN, J. 1999. Composites reinforced with cellulose based fibers. Prog. Polym. Sci., v. 24, 221-279.

CABALLERO, J. A.; FONT, R. MARCILLA, A. 1996. Comparative study of the pyrolysis of almond shells and they fractions, holocellulose and lignin. Thermochimimica Acta, v 276, 5777.

CHUNG, K. F.; YU, W. K. 2002. Mechanical properties of structural bamboo for bamboo scaffoldings. Engineering structures, v. 24, 14 p.

DEUS, J. F. D.; MONTEIRO, S. N.; ALMEIDA, J. R. M. 2005. Effect of drying, molding pressure, and strain rate on the flexural mechanical behavior of piassava (Attalea funifera Mart) fiber -polyester composites. Polymer Testing, v. 24, 6 p.

FRANCO, J. P. F. 2010. Aproveitamento da fibra de epicarpo do coco de babaçu em compósito com matriz de epóxi: estudo do efeito do tratamento da fibra. Dissertação de mestrado. UFRN. Programa de pós-graduação em ciências e engenharia de materiais.

GHAVAMI, K. 2005. Bamboo as reinforcement in structural concrete elements. In: Cement \& concrete composites, v.27, 637-649.

KONGKEAW, P.; NHUAPENG. W.; THAMAJAREE, W. 2011. The effect of fiber length on tensile properties of epoxy resin composites reinforced by the fibers of bamboo (Thyrsostachys Siamensis Gamble). Journal of the Microscopy Society of Thailand, v. 4, 46-48.

LIU D.; SONG J.; ANDERSON D. PCHANG P. R.; HUA Y. 2012. Bamboo fiber and its reinforced composites: structure and properties. Science+Business Media. Ed Springer.

MACHADO, G. O. 2000. Preparação e caracterização de CMC e CMC grafitizada. Dissertação de mestrado. Programa de mestrado em ciências e engenharias de materiais - Instituto de Quimica de São Carlos.

NOGUEIRA, F. M. 2009. Bambucon - bambu reforçado com microconcreto armado. Monografia - Curso de Especialização em Construção Civil da Escola de Engenharia. UFMG.

PEREIRA, M.A. 2001. Bambu: espécies, características e aplicações. Bauru - SP. Editora da UNESP, $58 \mathrm{p}$.

PERSSON, H SKARENDAHL, A. 1984. Natural Fibre Concrete for Roofing Sheets and Other Purposes in: SAREC report. Natural Fibre Concrete, SAREC Stockolm.

PHILIPP, P.; D’ALMEIDA, M. L. O. 1998. Celulose papel: Tecnologia de fabricação da pasta celulose. São Paulo $2^{\mathrm{a}}$ Ed., IPT., v1, pg. 48. 
REIS, E. G. 2013. Compósitos de fibras de taquara (Merostachys sp.) e matriz de poliéster e epóxi. Dissertação. (Mestrado em Ciências e Engenharia dos Materiais) - Setor de Tecnologia, Universidade do Estado de Santa Catarina.

XIE X.; ZHOU Z.; JIANG M.; XU X.; WANG Z.; HUI D. 2015. Cellulosic fibers from rice straw and bamboo used as reinforcement of cement-based composites for remarkably improving mechanical properties. Elsevier, Composites Part B 78, 153-161.

YUMING, Y.; CHAOMAO, H. 2010. CHINA'S BAMBOO: Culture, resources, cultivation, utilization. Published by International Network for Bamboo and Rattan (INBAR), Beijing, China. 